\title{
A Robust Design Methodology Suitable for Application to One-off Products
}

\author{
R. I. WHITFIELD, P. N. H. WRIGHT, G. COATES \& W. HILlS
}

SUMMARY Robust design is an activity of fundamental importance when designing large, complex, one-off engineering products. Work is described which is concerned with the application of the theory of design of experiments and stochastic optimization methods to explore and optimize at the concept design stage. The discussion begins with a description of state-of-the-art stochastic techniques and their application to robust design. The content then focuses on a generic methodology which is capable of manipulating design algorithms that can be used to describe a design concept. An example is presented, demonstrating the use of the system for the robust design of a catamaran with respect to seakeeping.

\section{Introduction}

A product's robustness is a measure of the variation in its utility experienced in a typical application. That is to say, the lower the sensitivity or variation in utility, the greater the robustness of the design. In this work, we consider robust design to be the process by which a design is produced in which changes in the selected variables which define the optimum design have relatively little effect on the performance of the design, i.e. the behaviour of the selected design is insensitive to modest changes in the variables. During the early stages of the design process, it is essential that many alternative proposals are examined in order to identify those designs which are robust. One of the difficulties encountered during this stage is that time is usually limited. In the case of large complex products, particularly those classified as made-to-order (MTO) or one-off products, this shortage of time causes a further complication since models which accurately represent the design and its behaviour or performance are, by necessity, large and complex. Under these circumstances, designers often resort to using concept design models which lack definition or decompose the complex model into a set of submodels. These submodels are then optimized and the overall compromise 'best design' is assumed to be defined by a combination of those variables and criteria which optimize the individual constituent and submodels.

This approach can be misleading and is flawed. A better approach is to seek methods which allow the full, complex model to be used but, by selecting a set of points in the design space, according to some prescribed strategy, a regression equation can be derived which accurately represents the response surface for the design space. This

Dr I. Whitfield, G. Coates and Professor W. Hills, Engineering Design Centre, and P. Wright, Department of Marine Technology, Newcastle University, Arm strong Building, Newcastle upon Tyne NE1 7RU, UK. Correspondence should be addressed to W. Hills. 
definition of the design space can then be searched to locate the global optimum design (once the surface has been defined). Search methods such as genetic algorithms (GAs) are particularly effective under such circumstances when the response surface may exhibit several local optima. Traditionally, engineers have sought to define their design models by representing them in the form of mathematical models and then exploring the surface using some systematic search technique. This usually involves very large numbers of evaluations of the model and the performance criteria. Box and Hunter [1] and Taguchi and Wu [2] suggested a method for determining the nature of the design space based on the design of experiments and statistical analysis. Lucas [3] extended this approach to achieve a robust process using response surface methodology. These techniques have been adopted as the basis for a new approach to 'robust design' which is particularly applicable to MTO product design.

\section{Background}

In the $1940 \mathrm{~s}$, Japanese industry recognized that, if it was to be competitive there was a critical need to improve the quality of the products it produced and the associated manufacturing design process. Genichi Taguchi, a quality consultant, was given the task of developing a methodology to meet these requirements. Consequently, robust design was established as a systematic methodology involving the application of statistical techniques to improve product quality and process design. Robust design improves product design quality and enables manufacture at low cost by making product and process performance 'robust'.

Subsequently, much research has been carried out to improve Taguchi's robust design methodology; particularly, the statistical techniques used. The aim was to improve product quality while making significant cost savings.

Taguchi's method for robust design is based on experimental design and statistical analysis. The approach to experimental design involves a product array which comprises a control array and a noise array. In an experiment, each combination of the control array is run with every combination of the noise array. Taguchi's robust design methodology is based on maximizing signal-to-noise (SN) ratios. The SN ratio, or quality characteristic, is typically given by

$$
\mathrm{S} / \mathrm{N}=-10 \log [\mathrm{MSD}]
$$

where MSD refers to the mean square deviation of the objective function. The quality characteristic is produced by differentiating design variables into control variables and signal variables. A robust optimum design is identified by locating the optimum values for the control variables to reduce variation and then adjusting the signal variables to shift the mean, achieved by maximizing the SN ratio.

Several important improvements to Taguchi's original work have been suggested by Chen et al. [4] who applied the methods to top-level design specifications for the airframe and propulsion system of a high-speed civil transport system. Welch et al. [5] further improve robust design methodology using combined arrays as opposed to Taguchi's product array. Other useful improvements have also been suggested by Engelund et al. [6] and Unal and Stanley [7].

\section{The Proposed Robust Design Methodology}

The robustness framework was produced using a similar methodology to that of Taguchi while using state-of-the-art statistical techniques to undertake the methodology. The work has been divided into two distinctive areas: to produce a robustness 


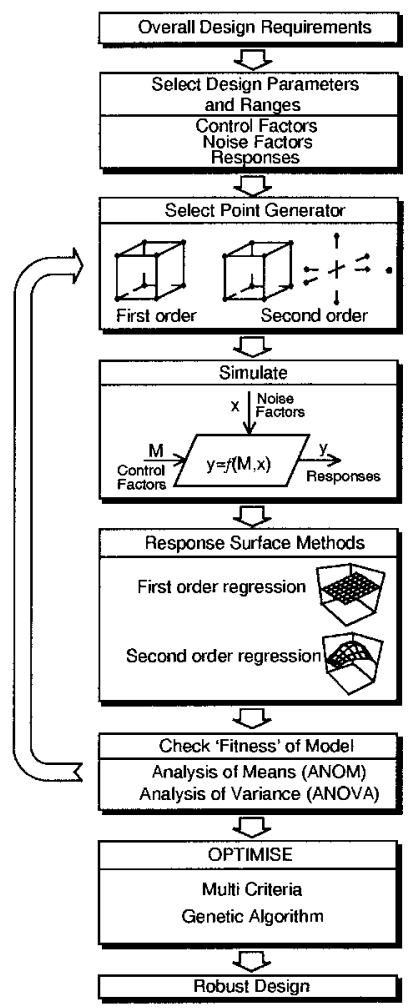

(a)

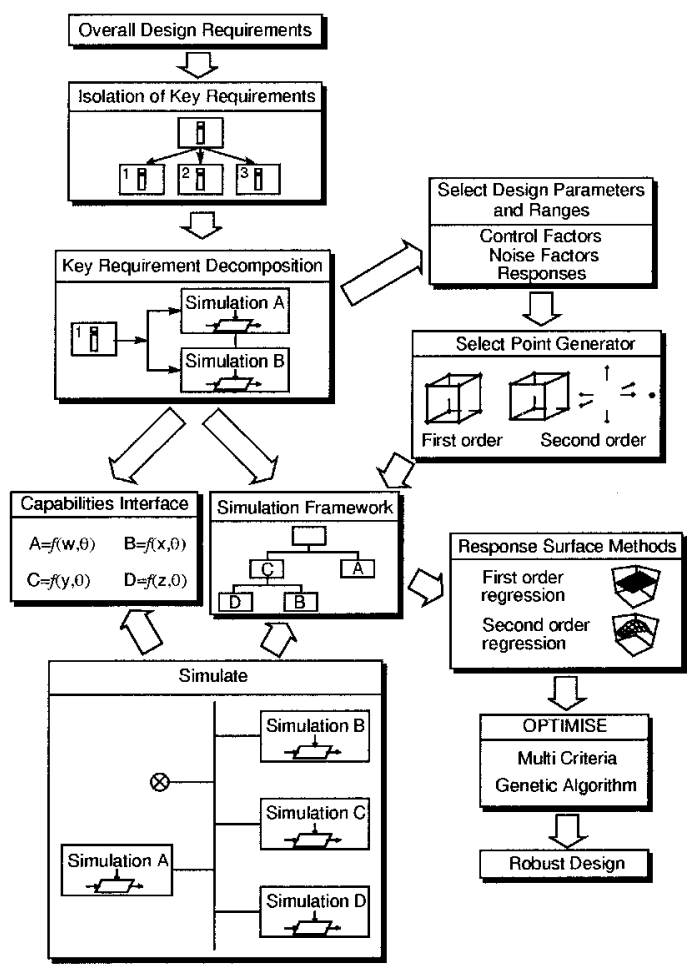

(b)

FIG. 1(a). Robustness framework; (b) design coordination system.

framework, and to allow for coordination during the design of robust MTO products. The robustness framework has been developed to a prototype stage to test the validity of the statistical techniques to the types of problems envisaged, while the design coordination system is in the early stages of development. Graphical representations of the robustness framework and design coordination system can be seen in Fig. 1. The robust design process presented within Fig. 1 has been automated using research software produced by the authors. This software is currently in the process of being utilized within several industrial applications.

\subsection{Robustness Framework}

The only strong connection remaining with the work of Taguchi is the philosophy of Taguchi's robust design and the parameterization of the design concept into controllable and uncontrollable design variables. The method is initiated with a statement of the goals to be achieved and the constraints to be satisfied. It is assumed at this point that there exists a means available to evaluate those requirements. Given a set of design algorithms capable of representing the design performance, the input and output parameters for each tool can be analyzed and selected based upon interpretations of their significance. The design space can now be controlled by selecting upper and lower bounds for the parameters and determining the type for each parameter, i.e. control, noise or response. 
Depending upon the stage of the analysis, a suitable point generator is selected using design of experiments (DOE) theory. The point generators available are full and variable fractional factorial and central composite design. The full and fractional factorial designs are available for any order of problem, whereas the central composite design is designed to be used for second-order problems only.

Typically, the first run of the analysis uses a saturated fractional factorial design to provide an overview of the design problem. With this information, variables can be removed from the analysis that are not considered to be significant with respect to the criteria. This technique relies upon the assumption that the main effects have greater significance than the interaction terms; hence, the removal of the variable from the analysis does not have any consequence on the response surface generated. This method allows the design to be studied without a great deal of information being required to define the design process. Subsequently, many variables may be added to the problem definition and hence into the analysis without dramatically increasing the size of the experiment. A second-order response surface design can then be used with the significant variables to produce an improved representation of the design concept.

The methodology involves the use of computer-based design algorithms; however, it is anticipated that experimental or full-scale test runs could be undertaken and data collected which could be utilized by the framework. Information is passed to the design algorithms which are then executed in the appropriate order to facilitate the correct flow of information. The responses are then taken from the output files and collated for use by the response surface module.

The control, noise and response variables are used to produce a set of normal equations. The normal equations can then be solved using a variety of different methods to produce a series of regression equations for the mean and variance of each response and constraint as functions of the input variables. Analysis of variance is then performed on the regression equations to check for 'goodness of fit'.

Currently, a single objective GA method is used to obtain an optimum design using the regression equation. The GA is preferred rather than more traditional hill-climbing techniques due to the ability of the DOE module to generate experiments of orders having local optima. The GA module, however, is obviously restrictive in its inability to deal with multiple objective functions and constrained problems. Research is nearing completion within the Newcastle Engineering Design Centre, at the University of Newcastle upon Tyne, on the use of a GA tool that enables multiple objectives and constraints to be considered, and it is intended that this tool will be incorporated for optimization purposes. Rather than producing an optimum design, the multi-criteria GA produces a pareto-optimal set of designs.

\subsection{Design Coordination System}

A design coordination system is currently being developed to facilitate the generation of robust designs within a concurrent and distributed computing environment. Within this system, a concurrent and distributed framework and an agent communication architecture are being developed [8]. The aim of the design coordination system is to enable design algorithms to be executed across a network and a variety of computer platforms. Given the overall design requirements, a suite of design algorithms will be available to enable some computation to be carried out. It is these design algorithms which need to be coordinated.

The design coordination system is the interface between the point generator and response surface methods embodied within the robustness framework. The point 
generator, which selects the design concepts to be evaluated, supplies the concepts to the design coordination system. Evaluated criteria produced by the design coordination system are collated and subsequently used by the robustness framework to construct response surfaces.

\section{Problem Description}

For development and evaluation purposes, a case study was selected with which staff in the Department of Marine Technology and the Engineering Design Centre have undertaken considerable work. The problem incorporates a single design algorithm which is capable of giving a number of measurements for the seakeeping of a catamaran. The objective of the work is to explore the design space for the catamaran and select a concept which is most robust with respect to selected seakeeping quantities at any particular waveheading.

\subsection{Design For Seakeeping Strategies}

For both monohulls and multihulls, there is a need to develop strategies which can clearly indicate how the designer may modify the hullform geometry so that improvements in the vessels' behaviour in waves can be realized. This must be done in such a manner that other design considerations such as calm water resistance and intact stability are not compromised. However, no general rules exist to advise the designer how a hullform may be modified to achieve specific improvements. The existence of such rules would necessitate in-depth 'cause and effect' understanding regarding changes in the hullform geometry parameters of significance and the various aspects of seakeeping which influence the performance of a vessel in waves, such as resultant motions and accelerations at specific locations, deck wetness and slamming. There is then a need to provide support for the designer at the conceptual design stage to allow either manual or automatic searches for optimal hullforms.

At Newcastle University, tools have been developed to aid the designer in identifying the necessary 'cause and effect' relationships required to improve design for seakeeping. Two approaches have been developed: 'forward analysis' and 'inverse analysis'. The forward analysis approach involves the production of 'design charts' which give a graphical indication of the degree of change experienced in any one of a number of seakeeping quantities as a function of selected primary and secondary hullform parameters, while maintaining the displacement and block coefficient such that the overall fullness of the underwater form is preserved. Primary parameters selected are length, $L$, breadth to draught ratio, $B / T$, and, for catamarans, the separation between the demihull centrelines, $H_{\mathrm{s}}$. Secondary parameters selected are the waterplane area coefficient, $C_{\mathrm{wp}}$, the longitudinal centre of flotation, LCF, and the longitudinal centre of buoyancy, LCB. $C_{\mathrm{wp}}$ is the ratio of waterplane area to the area of the enclosing rectangle as a product of length and breadth. LCF is the longitudinal position of the geometric centroid of the waterplane shape. Similarly, the LCB is the longitudinal position of the geometric centroid of the underwater geometry. These indicate the fullness of the waterplane, the centroid of the waterplane and the centroid of the underwater volume respectively. The necessary complex manipulation of the hullform to achieve practicable changes to these parameters has been automated to provide on-line manipulation of the hull lines as changes to these parameters are sought and the resulting hullform is evaluated. Hearn et al. [9] reported the forward analysis approach applied to catamaran ships. The inverse analysis approach allows automatic 
identification of either primary, secondary or both groups of parameters. For catamaran hullforms, this has been based on the application of a GA [10].

Evaluation of any selected motion quantity is demanding at the early stages of design when geometry information is not finalized and large numbers of alternatives are likely to be examined. The normal frequency domain approach is to provide solutions of motion amplitudes, $s_{i}$, for motion responses in a number of sine waves of unit amplitude but differing frequency, $\omega$, for a particular wave heading relative to the vessel, $\phi$, to provide a response amplitude operator (RAO) for each motion quantity desired, $H\left(\omega_{\mathrm{e}}, \phi\right)$. The rigid body motions of particular interest are heave $(i=3)$, roll $(i=4)$ and pitch $(i=5)$. In conjunction with an encountered wave spectrum representing the irregular sea state of interest, $\Gamma_{\omega}\left(\omega_{\mathrm{e}}, \phi\right)$, this allows an appropriate response spectrum to be calculated, $\Gamma_{\mathrm{r}}\left(\omega_{\mathrm{e}}, \phi\right)$, from the area of which root mean square (RMS) values of response spectrum can be calculated.

$$
\Gamma_{\mathrm{r}}\left(\omega_{\mathrm{e}}, \phi\right)=\left(\left[H\left(\omega_{\mathrm{e}}, \phi\right)\right]^{2} \cdot \Gamma_{\omega}\left(\omega_{\mathrm{e}}, \phi\right)\right)
$$

To improve motion characteristics, the need is then to minimize the area under the response spectrum. To reduce the effort required, the Newcastle approach to seeking improved responses is to minimize the peak and area of the RAO, $H\left(\omega_{\mathrm{e}}, \phi\right)$, rather than the area of the response spectrum, $\Gamma_{\mathrm{r}}\left(\omega_{\mathrm{e}}, \phi\right)$, directly, although the effect is the same and will provide improvement for any selected sea state while also reducing the level of calculation required for evaluating each alternative.

In order to provide the necessary RAO evaluations, the strip theory of Salvesen et al. [11] is utilized. The explicit calculation of the necessary reactive hydrodynamic coefficients and excitation forces for each alternative hullform to provide the necessary solution for each RAO would nevertheless be complex and demanding in terms of computational effort and inappropriate at the earliest stages of design. In order to allow complex evaluation of the alternative hullforms, but with considerably reduced effort, uniquely, the required hydrodynamic data has been pre-calculated for a series of generalized two-dimensional ship sections. The coefficients appropriate to each alternative hullform are then found by mapping these stored solutions to the sections under investigation, which are defined from a three-dimensional model of the particular underwater surface of the hull geometry being investigated. These two-dimensional values are then integrated over length to provide global coefficients. This approach allows the necessary complex evaluation to be achieved for each alternative hullform with a high degree of accuracy in the order of one-thirtieth of the time to undertake the explicit calculation of the hydrodynamic coefficients [9]. This ability to provide a fast accurate design algorithm becomes even more important for catamaran design. For monohulls, improved motions in head seas are accepted to give improvement at other wave headings, but for catamarans, the sensitivities to hullform geometry are more complex and the influence on roll and vertical motions across oblique wave headings needs to be explicitly considered. The development of this approach is described by Hearn et al. [10,12] along with its validation [13].

This approach to evaluate the performance of designs investigated is required because empirically derived relationships to relate changes in geometry and motion characteristics are not satisfactory. The cause and effect relationships are particular to each ship type being investigated and show particular sensitivity in the case of catamaran design. However, previous experimental studies as well as empirical studies have validated this approach.

Previous attempts to identify optimal hullforms via the application of a GA have been in head seas $\left(180^{\circ}\right)$ and beam seas $\left(90^{\circ}\right)$ in order to identify conflicts in beneficial 


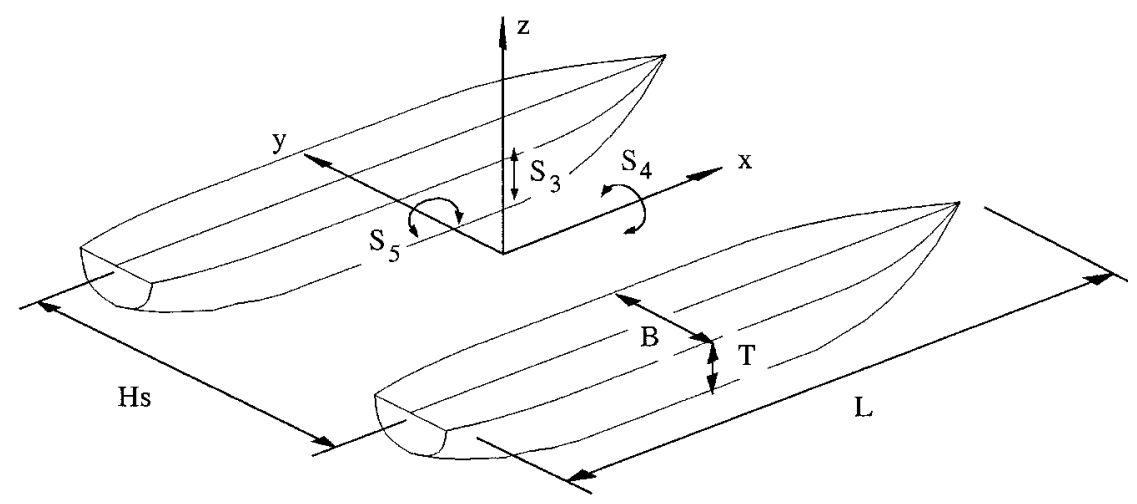

FIG. 2. Design variables for a catamaran.

hullform changes for heave, pitch and roll independently [10]. The relative motion as a function of all three motions at a waveheading of $135^{\circ}$ was one quantity investigated by Hearn et al. [12] in order to reduce the problematic catamaran phenomena of 'corkscrewing', where pitch and roll motions combine. More recently, rather than just including resistance performance as a constraint, it was included in a number of multiple criteria objective functions based on resistance and motion quantities in Hearn and Wright [14]. The application of a Taguchi-based approach allowed the motions of heave, pitch, roll and relative motion, as a function of all three constraints to be assessed over a number of waveheadings in order to identify a robust optimal form [15]. Here, this approach is developed further with the application of the robustness framework presented in this paper.

\subsection{Catamaran Design Space}

In keeping with earlier work on seakeeping for design, six controllable design variables were used to define a basic catamaran design concept.

- Primary design variables:

- Secondary design variables:

- Noise variable:
Hull length, $L$;

Breadth to draught ratio, $B / T$;

Distance between demihull centres, $H_{\mathrm{s}}$;

Longitudinal centre of buoyancy, LCB;

Coefficient of waterplane, $C_{\mathrm{wp}}$,

Longitudinal centre of floatation, LCF, Waveheading, $\phi$.

The seakeeping quantities selected here to be minimized are the peak values of the RAOs associated with heave, roll, pitch and the relative motion at the bow of each demihull, as a function of all three motion quantities combined relative to the free surface elevation, $\zeta_{x, y}$, at the bow located at $(x, y)$.

$$
s_{\mathrm{r}}=s_{3}+y \cdot s_{4}-x \cdot s_{5}-\zeta_{x, y}
$$

Criteria: maximum heave amplitude, $\left|s_{3}\right|_{\max } ;$ maximum roll amplitude, $\left|s_{4}\right|_{\max } ;$ maximum pitch amplitude, $\left|s_{5}\right|_{\text {max }}$; maximum relative bow motion, (RBM), $\left|s_{\mathrm{r}}\right|_{\max }$. A diagrammatic representation of the design variables and criteria can be seen in Fig. 2.

Due to the restrictions imposed by the optimization method, and to keep the 
R. I. Whitfield et al.

TABLE I. Design space for catamaran problem

\begin{tabular}{llccccc}
\hline Variable & Type & Parent & Lower Limit & Mid Point & Upper Limit & Symbol \\
\hline$\phi$ & Noise & & 90 & 135 & 180 & $x_{1}$ \\
$L$ & Control & $104.0 \mathrm{~m}$ & $-9 \%$ & $+1 \%$ & $+11 \%$ & $x_{2}$ \\
$B / T$ & Control & 2.0 & $-9 \%$ & $+1 \%$ & $+11 \%$ & $x_{3}$ \\
$H_{\mathrm{s}}$ & Control & $31.0 \mathrm{~m}$ & $-9 \%$ & $+1 \%$ & $11 \%$ & $x_{4}$ \\
LCB & Control & 45.408 & $-0.9 \%$ & $+0.1 \%$ & $+1.1 \%$ & $x_{5}$ \\
$C_{\text {wp }}$ & Control & 0.758 & $-0.9 \%$ & $+0.1 \%$ & $+1.1 \%$ & $x_{6}$ \\
LCF & Control & 43.306 & $-0.9 \%$ & $+0.1 \%$ & $+1.1 \%$ & $x_{7}$ \\
RBM & Response & & - & - & - & $Y$ \\
\hline
\end{tabular}

problem relatively simple, the objective function used was the relative bow motion and was constrained only by the upper and lower bounds of the design space. This is justified as other investigations of the same hullform have demonstrated equal or better resistance performance for changes made to improve these motion quantities. The robust design methodology, however, allows for multiple objective functions and multiple constraints.

The design space was explored relative to a parent design and was expressed as a percentage change for the primary and secondary variables and in absolute terms for the noise variable as shown in Table I.

\subsection{Experimental Results}

A resolution three fractional factorial design was used for a screening run to establish the relative importance of each of the design variables. The following regression equation was obtained using eight experimental runs of the design algorithm.

$$
Y=0.96-0.0003 \cdot x_{1}-0.28 \cdot x_{2}-0.3 \cdot x_{3}-0.024 \cdot x_{4}-0.22 \cdot x_{5}-6.18 \cdot x_{6}+5.93 \cdot x_{7}
$$

The upper limit for each variable was used to calculate the significance with respect to the relative bow motion for each variable and can be seen in absolute terms in Fig. 3 .

It is apparent from Fig. 3 that the relative bow motion obtained using a first-order analysis is dominated by $L, B / T, C_{\mathrm{wp}}$ and LCF; however, $H_{\mathrm{s}}$ and LCB were not considered to be sufficiently small to remove them from the analysis.

\section{R.B.M.}

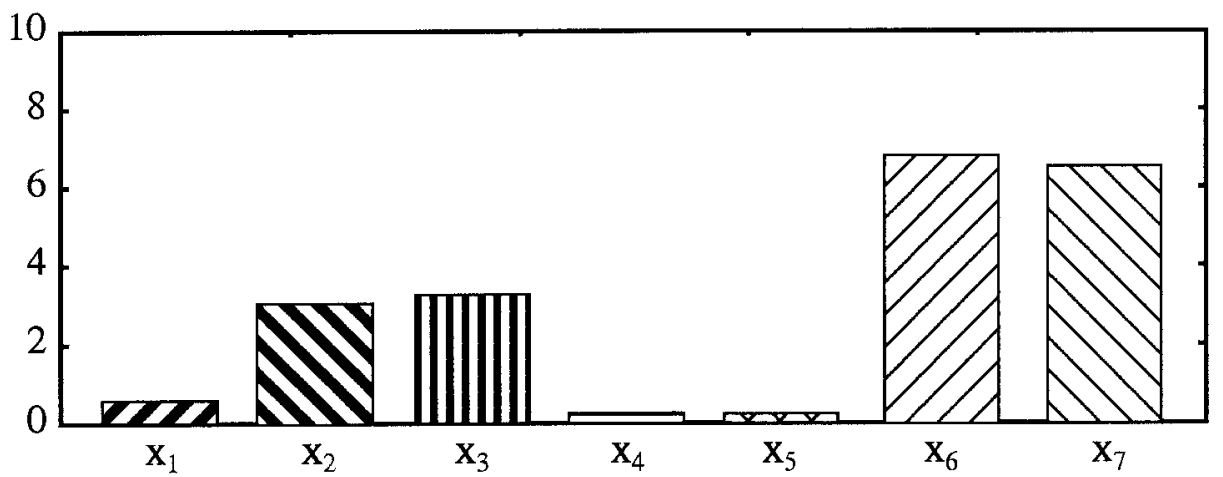

FIG. 3. Contribution to RBM of each design variable using first-order regression. 


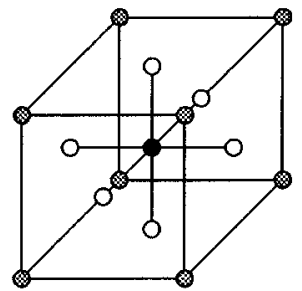

Face-Centred Cube
- Centre Point

- Factorial Points

O Star Points

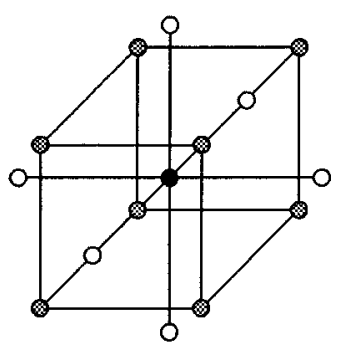

$\alpha= \pm 1$

$\alpha=\sqrt{k}$

FIG. 4. Representation of design points for central composite design.

A number of different second-order experimental plans were created and tested using the DOE module including full factorial, fractional factorial and central composite design (CCD). The CCD was created using both full factorial and resolution five fractional factorial designs for the first-order design. It was discovered that the CCD produced the most accurate representation of the design concept with the fractional factorial CCD producing the regression equation in 47 experimental runs, as opposed to 2187 experimental runs required for the full factorial design. The type of CCD used in this analysis is known as the face-centred cube and can be seen in Fig. 4. The experimental design consists of a first-order fractional or full factorial design augmented with star and centre points. The star point distance, $\alpha$, is given a value to allow the designer to achieve certain design properties. In this instance, $\alpha$ is given a value such that the factorial part defines the region contained within the design space. For comparison, Fig. 4 also shows a CCD having a star point distance greater than the factorial design space. The experimental plan was then used with the design algorithm to obtain a series of design concepts. The design space was subsequently explored in approximately $20 \mathrm{~min}$.

A set of normal equations were produced using the method of least squares based upon the information generated from the second-order analysis. These normal equations were then solved using Cholesky LU factorization to produce the following regression equation for the relative bow motion as a function of the seven design variables obtained using the CCD.

$$
\begin{aligned}
Y= & 3.6469-0.0786 x_{1}+1.1386 x_{2}+0.6558 x_{3}-0.0876 x_{4}+0.5590 x_{5}+2.5014 x_{6} \\
& -9.5733 x_{7}+0.0003 x_{1}^{2}-0.0107 x_{1} x_{2}-0.0073 x_{1} x_{3}+0.0005 x_{1} x_{4}-0.0051 x_{1} x_{5} \\
& -0.0283 x_{1} x_{6}+0.1020 x_{1} x_{7}-0.0007 x_{2}^{2}+0.0025 x_{2} x_{3}-0.0002 x_{2} x_{4}-0.0361 x_{2} x_{5} \\
& -0.0094 x_{2} x_{6}+0.0176 x_{2} x_{7}+0.0070 x_{3}^{2}-0.0006 x_{3} x_{4}-0.0165 x_{3} x_{5}+0.0018 x_{3} x_{6} \\
& -0.0175 x_{3} x_{7}-0.0013 x_{4}^{2}-0.0041 x_{4} x_{5}-0.0077 x_{4} x_{6}+0.0013 x_{4} x_{7}-0.0814 x_{5}^{2} \\
& -0.0375 x_{5} x_{6}+0.2128 x_{5} x_{7}+0.0273 x_{6}^{2}+0.7284 x_{6} x_{7}+0.1844 x_{7}^{2}
\end{aligned}
$$

This regression equation is generated and used automatically within the robustness framework and, hence, does not allow the removal of any terms. Comparisons were made between a number of randomly selected points within the design space using both the regression equation and the design algorithm. Good agreement was obtained and, hence, it was decided that the regression equation was a suitable representation of the design concept and could be used for further evaluation and optimization purposes. The regression equation was subsequently used to produce the response surfaces seen 

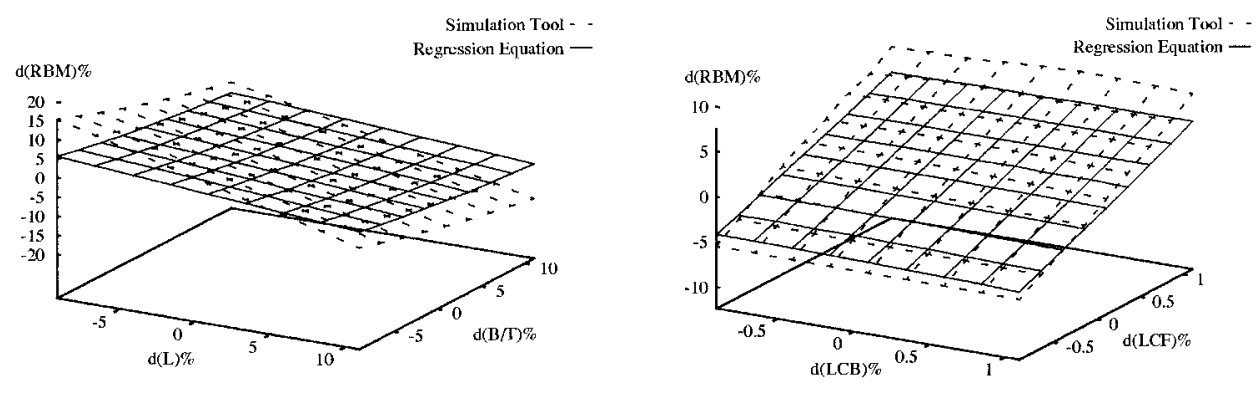

FIG. 5. RBM primary and secondary design charts.

in Fig. 5 for a waveheading of $135^{\circ}$. RBM was plotted against hull length and breadth to draft ratio for the parent $H_{\mathrm{s}}$, as well as LCF and LCB for the parent $C_{\mathrm{wp}}$. The values of RBM are obtained with respect to the parent design, hence increasing negative values indicate increasing desirability. The design algorithm was used to obtain results from the same design points for comparison. The designer could manually select an optimal combination of hullform parameters based on the optimal individual combinations of primary and secondary parameters indicated from the primary and secondary design charts. This superposition of the optimal solution for each group has been found previously to provide an indication of the global optimum, although not necessarily the optimal value, for each parameter.

Based on this approach, the design charts would indicate that the optimum design for reducing the RBM for the parent values of $H_{\mathrm{s}}$ and $C_{\mathrm{wp}}$, at a waveheading of $135^{\circ}$, would be to maximize $L$ and $B / T$, and move LCF forward and LCB aft, although LCB was again shown to be of little significance with respect to RBM.

The GA was then used to search the design space for the optimum design. Since the GA was only capable of optimizing a single objective function, the objective function used comprised the summation of the RBM at seven waveheadings at $15^{\circ}$ intervals. The GA was used initially to optimize the regression equations using the design space given in Table I. The optimum design was obtained in approximately $5 \mathrm{~min}$. For comparison, the optimization was repeated using the design algorithm instead of the regression equation, again using the design space defined in Table I. This optimization process was completed in approximately $8 \mathrm{~h}$. Finally, a more traditional Taguchi-type approach was used to determine the optimum design based upon the primary parameters, using the same methodology as that used by Sen et al. [15]. The results from these optimization processes can be seen in Table II.

For these optimum designs, the design algorithm was then used to determine the RBM across the range of waveheadings (Fig. 6). It is apparent that the optimum design obtained using the regression equation produces a greater reduction in RBM across the waveheadings than that using both the design algorithm and the method chosen by Sen

TABLE II. Design variable values selected by GA

\begin{tabular}{lcccccc}
\hline Method & $\mathrm{d}(L) \%$ & $\mathrm{~d}(B / T) \%$ & $\mathrm{~d}\left(H_{\mathrm{s}}\right) \%$ & $\mathrm{~d}(\mathrm{LCB}) \% L$ & $\mathrm{~d}\left(C_{\mathrm{wp}}\right) \%$ & $\mathrm{~d}(\mathrm{LCF}) \% L$ \\
\hline Regression equation & 7.69 & 10.70 & 10.50 & $0.858_{\text {aft }}$ & 1.10 & $0.894_{\text {forward }}$ \\
Simulation tool & 10.9 & 9.73 & 11.0 & $0.25_{\text {forward }}$ & 0.893 & $0.827_{\text {forward }}$ \\
Sen method & 10.0 & 10.0 & 10.0 & - & - & - \\
\hline
\end{tabular}




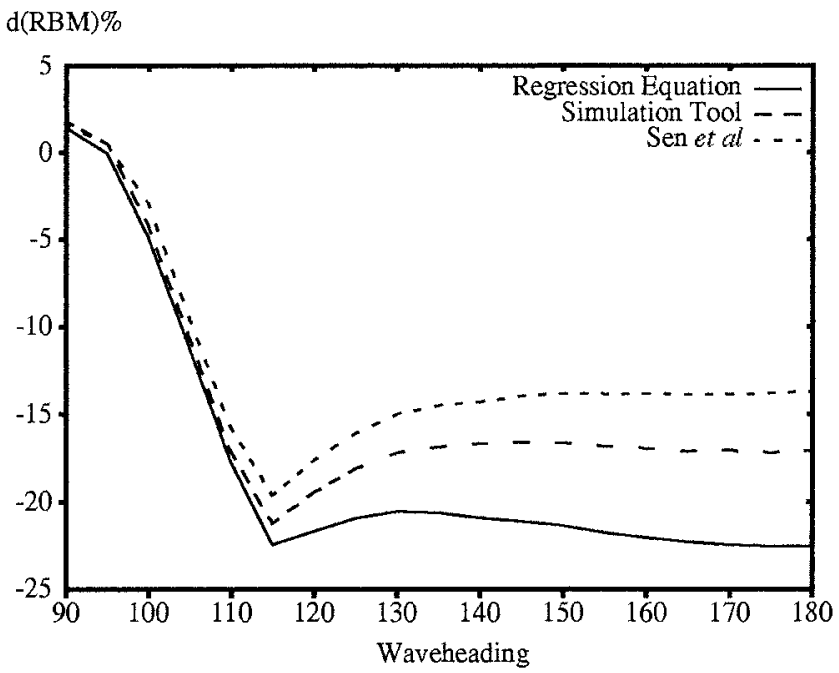

FIG. 6. Variation in RBM with waveheading.

et al. [15]. It is also clear that significant improvements were obtained with an average reduction in $\mathrm{RBM}$ of approximately $18 \%$ across all waveheadings, as opposed to a $12 \%$ reduction in RBM achieved by Sen et al. It was apparent that neither the optimum derived using the regression equation, the design algorithm or that found using the Taguchi approach, reduced the RBM at waveheadings around $90^{\circ}$.

The GA was then used to identify the optimum design at $90^{\circ}$ using both the regression equation and design algorithm. Similar results were obtained using the two methods and indicated that the optimum design for a waveheading of $90^{\circ}$ lies in a completely different region of the design space than that located for the range of waveheadings.

Finally, the analysis was repeated using heave, pitch and roll separately as the objective functions. The optimum designs were again compared with results obtained using the Taguchi methodology. The design algorithm was again used to obtain values for heave, pitch and roll for the optimum designs obtained using both methodologies.

These results can be seen in Figs. 7-9, having average reductions in the peak amplitudes of the heave, pitch and roll RAOs of $33 \%, 29 \%$ and $25 \%$ respectively using the proposed methodology. This compares favourably with the reductions of $20 \%, 23 \%$ and $23 \%$ for heave pitch and roll obtained using the Taguchi methodology of Sen et al. [15].

\section{Discussion of Results}

The results of this work are consistent with the earlier cited references. The observation that $L, B / T$ and LCF are dominant within the design space investigated is consistent with earlier results where design charts were used to develop such cause and effect understanding. This dominance is again demonstrated in the design charts presented here (Fig. 5) and in the contribution to the regression equation shown in Fig. 3. The primary and secondary parameter changes indicated in Fig. 5 are consistent with previous findings. The primary design chart indicates that an increase in $L$ and $B / T$ are of benefit. Although not investigated for this comparison of the simulation and regression tools, these changes accompanied by a reduction in $H_{\mathrm{s}}$ would probably be of 


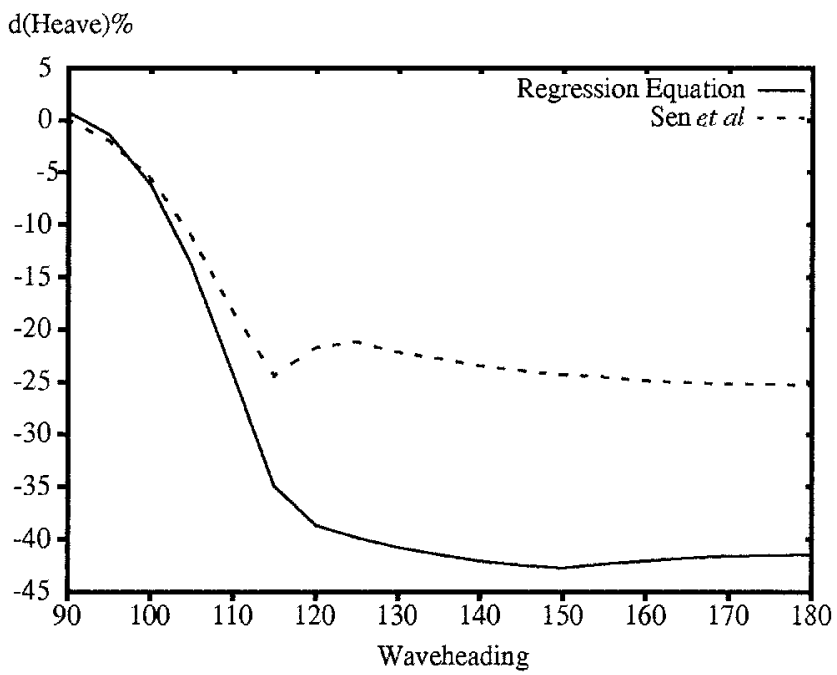

FIG. 7. Variation in heave with waveheading.

further benefit in reducing $\mathrm{RBM}$ for a waveheading of $135^{\circ}$. For the secondary parameter design chart in Fig. 5, the conclusion of moving LCF forward and LCB aft, subject to the parent $C_{\mathrm{wp}}$, would demonstrate further improvement if $C_{\mathrm{wp}}$ were increased.

The approach adopted has allowed significant improvement to be achieved in RBM across a wide range of waveheadings, as demonstrated by Fig. 6 . The lack of improvement for waveheadings approaching $90^{\circ}$ is explained by the dominance of the roll component over the vertical component motions of heave and pitch. Parameter changes of benefit to vertical motions and roll tend to conflict and occupy different portions of the search space, particularly with respect to $B / T$ and $C_{\text {wp }}$, which does not aid the identification of a compromise solution.

The primary parameter changes suggested to benefit $\mathrm{RBM}$ are consistent for the

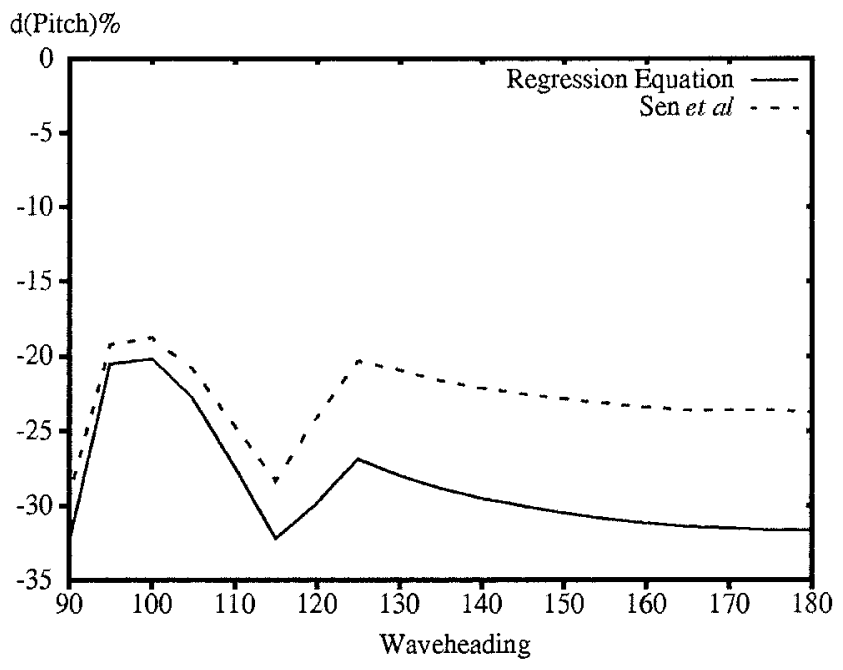

FIG. 8. Variation in pitch with waveheading. 


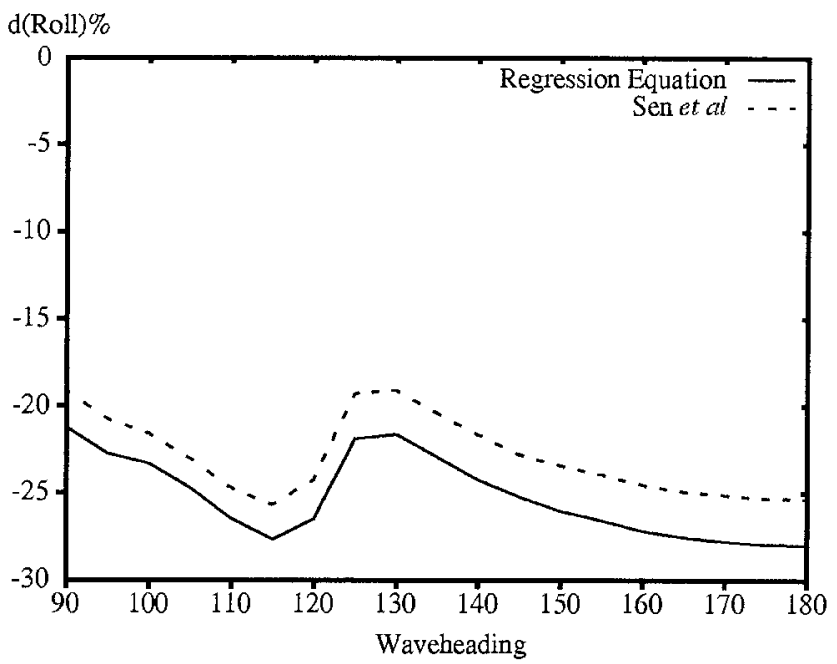

FIG. 9. Variation in roll with waveheading.

three methods investigated, as well as the best compromising solution found previously [15]. The secondary parameter changes suggested both by the regression equation and the design algorithm directly are also consistent with the exception of the LCB position indicated. The secondary changes given by the regression equation based method are recognizable from earlier work and are particularly beneficial in reducing vertical motions in head seas $[9,10]$, whereas the changes found by direct application of the design algorithm differ with respect to LCB. The optimum found using the design algorithm with LCB moving forward of the parent position is comparable to the compromise solution identified by Sen et al. [15] and earlier work where such a change was found to benefit pitch. The optimum identified from the regression equation would be preferred because of this consistency with earlier findings in reducing vertical motions, particularly in head seas where limiting responses such as slamming due to excessive relative motion at the bow result in loss of performance in waves due to the necessity to involuntarily reduce speed.

Although there is a relative insensitivity to LCB over the design space investigated, the difference in LCB position indicated from the application of the regression- and simulation-based optimization must be the major factor in their relative performance as optima in comparison to each other. The further improvement found by moving it aft, as indicated by the regression-based optimum, also substantiates the previous preference for this optima. However, if other considerations dependent on LCB, such as the trim of the vessel, become important, the results also demonstrate that it could be moved forward without incurring too large a penalty. The difference in LCB selection might be explained by the regression equation; in this case, providing a more well defined optima in this region than would be demonstrated by the design algorithm directly, with the result that when optimizing with the design algorithm explicitly, this optimum is not identified.

The results demonstrate that significant motion improvements can be found for several motion quantities simultaneously, through identifying appropriate combinations of hullform parameters via the approach presented. Although not presented, the RBM optimized form has been demonstrated to also have improved motion characteristics for heave, pitch and roll. The changes which result to the hullform geometry as a 
consequence of these modifications to the primary and secondary parameters can be rationalized by considering the individual geometry of the hull sections, the influence on the hydrodynamic coefficients and the overall consequence on the governing equations of motion and hence, the resulting change to the RAOs.

To provide further comparison with the Sen et al. approach, the peak heave, roll and pitch were also selected as individual objective functions and improved solutions were again found over the entire range of waveheadings considered.

The results obtained indicate that significant improvements in all aspects of performance of the catamaran can be obtained in considerably less time than with other optimization methods. The entire process of concept exploration and optimization using this methodology took approximately $30 \mathrm{~min}$, as opposed to $8 \mathrm{~h}$ using the design algorithm only. Execution times were obtained using a Sun UltraSparc platform. The design algorithm is configured to allow other optimization methods to be used, which would reduce the time taken to obtain the optimum. While the more traditional Taguchi approach achieved reductions in time to optimize compared with using the design algorithm, the approach was neither as fast as the proposed methodology nor did it produce as significant an improvement in performance. The reason for this was due to the differences in the methods of selection of the optimum designs. The Taguchi approach of Sen et al. explored the design space at discrete points using a full factorial experimental plan and selected the optimum design as the point which achieved the most desirable $\mathrm{SN}$ ratio. The proposed methodology explored the design space in a similar manner using a more sophisticated and efficient experimental plan to achieve a continuous representation of the design space which was then used for optimization purposes. Given this continuous representation, points anywhere within the design space can be selected and checked for optimality rather than the discrete points of the exploration stage.

\section{Conclusions}

The approach to robust design described in this paper has been shown to be efficient and effective when applied to a design problem in which the design model is complex and solutions computationally time consuming. Such models are common in the MTO field and further work is currently being carried out to determine the range of applicability of the proposed robust design methodology.

Experience indicates that further improvement in efficiency can be achieved by incorporating a multi-criteria approach including an appropriate GA and design selection technique. This approach is currently being investigated and early results indicate that significant improvements can be achieved.

Additional advantages of integrating the multicriteria GA and decision-making tools within this framework are that the pareto-optimal set will consist of designs which have each objective function and constraint expressed in terms of both the mean and variance. The designer will then have the ability to trade-off designs which have particular aspects of their performance that are on a flat region of the response surface to designs whose performance is on a more peaked region.

The software environment described in this paper has been developed by staff in the Newcastle Engineering Design Centre. There is comprehensive user documentation which guides the user through the methodology and application. This is essential since the focus of the Engineering Design Centre's programme is to encourage the use of such systems by collaborating industrial partners. Training on the system is provided by the development team for industry-based staff and other visiting researchers. 


\section{REFERENCES}

[1] Box, G.E.P. \& Hunter, J.S. (1961) The 2(k-p) fractional factorial designs. Part 1, Technometrics, 3, pp. 311-352.

[2] Taguchi, G. \& Wu, Y. (1980) Introduction to Off-Line Quality Control (Nagaya, Japan, Central Japan Quality Control Association).

[3] LuCAs, J.M. (1994) How to achieve a robust process using response surface methodology, fournal of Quality Technology, 26, pp. 248-260.

[4] Chen, W., Allen, J.K., Mavris, D.N. \& Mistree, F. (1996) A concept exploration method for determining robust top-level specifications, Engineering Optimization, 26, pp. 137-158.

[5] Welch, W.J., YU, T.K., Kang, S.M. \& SACKS, J. (1990) Computer experiments for quality control by parameter design, fournal of Quality Technology, 22, pp. 15-22.

[6] Engelund, W.C., Stanley, D.O., Lepsch, R.A., McMillian M.M. \& Unal, R. (1993) Aerodynamic configuration design using response surface methodology analysis, AIAA Aircraft Design Systems Meeting, August 1993, Monterey, California, pp. 1-11.

[7] Unal, R. \& Stanley, D.O. (1993) Designing for quality using response surface methods, American Society for Engineering Management 14th Annual Conference, October 1993, Dallas, Texas, pp. 7-11.

[8] Coates, G., Whitfield, R.I., Duffy, A.H.B. \& Hills, W. (1998) A design coordination methodology, Internal Report, Newcastle Engineering Design Centre.

[9] Hearn, G.E., Wright, P.N.H. \& Hills, W. (1994) Seakeeping for design: the demands of multihulls in comparison to monohulls, in: BrodDa, J. \& JoHANSSAN (Eds) Proceedings ICCAS '94, 8th International Conference on Computer Applications in Shipbuilding, 5-9 September 1994, Bremen, Germany, Vol. 2, pp. 11.12511.144 (Sweden, Berry Rasmnssan Reklam A B).

[10] Hearn, G.E., Wright, P.N.H. \& Hills, W. (1995) Seakeeping for design: development and application of an inverse analysis design methodology to multihull forms, RINA International Conference on Seakeeping and Weather, Paper No. 13, 28 February-1 March 1995, London, UK, 23 pp.

[11] Salvesen, N., Tuck, E.O. \& Faltinsen, O. (1970) Ship motions and sea loads, Transactions SNAME, 78, pp. 250-286.

[12] Hearn, G.E., Wright, P.N.H. \& Hills, W. (1995) Seakeeping for design: balancing the vertical and horizontal motions of a catamaran, Proceedings FAST'95, 3rd International Conference on Fast Sea Transportation, 25-27 September 1995, Lübeck-Travemünde, Germany, Vol. 1, pp. 205-220.

[13] Hearn, G.E., Wright, P.N.H. \& YaAkob, O.B. (1995) Seakeeping for design: identification of hydrodynamically optimal hull forms for large high speed catamarans, RINA, Proceedings of International Symposium On High Speed Vessels for Transport and Defence, Paper No. 4, 23-24 November 1995, 15 pp.

[14] Hearn, G.E. \& Wright, P.N.H. (1997) Seakeeping for design: optimisation of motion responses and wave making resistance of catamarans via the application of a genetic algorithm, Proceedings FAST'97, 4th International Conference on Fast Sea Transportation, 21-23 July 1997, Sydney, Australia, Vol. 1, pp. 231-240.

[15] Sen, P., RAO, Z. \& WRIGHT, P.N.H. (1997) Multicriteria robust optimization of engineering design systems under uncertainty, Proceedings ICED'97, 11th International Conference on Engineering Design, 19-21 August 1997, Tampere, Finland, Vol. 2, pp. 357-364. 